\title{
QUALIDADE DE VIDA DO ENFERMEIRO NO TRABALHO DOCENTE: ESTUDO COM O WHOQOL-BREF
}

\author{
Quality of life for nurses when teaching professionaly: a study with the Whoqol-bref \\ Calidad de vida del enfermero docente: estudio con el Whoqol-bref
}

Maria Rodrigues da Conceição ${ }^{1}$

Ângela Maria Alves e Souza ${ }^{4}$
Maria Suêuda Costa²

Maria Beatriz de Paula Tavares Cavalcante ${ }^{5}$
Maria Irismar de Almeida ${ }^{3}$

Maria Dalva Santos Alves ${ }^{6}$

\section{RESUMO}

0 estudo objetivou verificar a qualidade de vida do enfermeiro docente que trabalha em instituição federal, estadual e privada e comparar os resultados entre os três grupos. Os dados foram obtidos por meio do Whoqol-bref, com 26 perguntas, e aprovado pelo Comitê de Ética e Pesquisa. Foram 38 professores doutores, com exercício da docência na graduação e pós-graduação. Destes, 30 eram casados; seis, solteiros; e dois, separados, com média de idade de 46,5 anos. Quanto à qualidade de vida global, somente os professores da universidade privada têm minoria no escore "bom", mas nos domínios físico e psicológico a soma dos percentuais dos escores não apresentou diferenças relevantes, pois no primeiro foi de $47 \%$ e no segundo 43,4\%; entretanto, para os domínios das relações sociais e do meio ambiente, foram 22,2\% e 30,6\%. Utilizar o Whoqol-bref, mesmo com amostra reduzida, forneceu informações relevantes sobre a qualidade de vida docente.

Palavras-chave: Qualidade de vida. Docente. Trabalho. Enfermagem.

\begin{abstract}
The study focused in verifying the quality of life of the nurse teacher that works in a federal, municipal and private institution, comparing the results among the three groups. Data were obtained through the Whoqol-bref, using 26 questions approved by the Ethics and Research Committee. There were 38 doctorate professors, to carry out teaching at the undergraduate and graduate program. From these, 30 were married, 06 singles and 02 divorced, with an average age of 46.5 years. Concerning the overall quality of life only teachers from private university, the minority, have good scores, but in the physical and psychological areas the percentage summary of the scores showed no significant differences, in the first was $47 \%$ and $43.4 \%$ in the second, however for the domains of social relationships and environment, were $22.2 \%$ and $30.6 \%$. Using the Whogol - bref, even with a reduced sample, it provided relevant information about the teaching of quality of life.
\end{abstract}

Keywords: Quality of life. Faculty. Work. Nursing.
Resumen

El objetivo fue verificar la calidad de vida del enfermero maestro de institución federal, estadual y particular y comparar los resultados entre los tres grupos. Los datos fueron obtenidos a través del Whoqol - bref, con 26 preguntas y aprobado por el Comité de Ética en Investigación. Fueron 38 maestros doctores, con enseñanza en el pregrado y postgrado. De estos, 30 eran casados, seis solteros y dos separados, con edad media de 46,5 años. Cuanto a la calidad de vida global solamente los profesores de la universidad privada presentaran reducidos escores - buena salud - pero en los dominios físico y psicológico la suma de las puntuaciones no mostraron diferencias significativas, con $47 \%$ y $43.4 \%$. Sin embargo, para los dominios de las relaciones sociales y el medio ambiente, fueron $22,2 \%$ y $30,6 \%$. El uso del Whoqol - bref, incluso con una pequeña muestra proporcionó información relevante sobre la calidad de la enseñanza de la vida.

Palabras-clave: Calidad de vida. Docentes. Trabajo. Enfermería.

${ }^{1}$ Enfermeira. Especialista em Enfermagem do Trabalho. Mestre em Enfermagem pela Universidade Federal do Ceará. Fortaleza-CE. Brasil. E-mail: mariarocon@ig.com.br; ${ }^{2}$ Enfermeira. Doutora em Enfermagem. Auditora da Secretaria de Saúde do Município de Fortaleza- CE. Brasil. E-mail: sueudacosta@yahoo.com.br; ${ }^{3}$ Enfermeira. Doutora em Enfermagem. Professora Adjunta IV da Universidade Estadual do Ceará. Fortaleza - CE. Brasil. E-mail: irismaruece@gmail.com; ${ }^{4}$ Enfermeira. Doutora em Enfermagem. Professora Adjunta IV da Universidade Federal do Ceará. Fortaleza - CE. Brasil. E-mail: amas@ufc.br; ${ }^{5}$ Enfermeira. Mestre em Enfermagem. Enfermeira do Hospital Monte Klinikum. Fortaleza - CE. Brasil. E-mail: beatriz_cavalcante@hotmail.com ; ${ }^{6}$ Enfermeira. Doutora em Enfermagem. Professora Associada II da Universidade Federal do Ceará. Fortaleza - CE. Brasil. E-mail: dalva@ufc.br 


\section{INTRODUÇÃO}

Estudos sobre a Enfermagem no contexto hospitalar acentuam que o trabalho em saúde é hoje considerado um processo estabelecido no contexto produtivo, no qual o homem participa como agente, podendo constituir-se como fator determinante para o desgaste da saúde dos trabalhadores ${ }^{1}$. Os padrões de morbidade/mortalidade apresentam-se de acordo com a maneira como inseridos nas formas de produção capitalista, e o magistério também recebe estas influências.

A saúde do trabalhador, até daquele que cuida da população, parece interessar pouco, o que é comprovado pela escassez de estudos. Um especialista nos assuntos da educação brasileira defende com muita propriedade a ideia de que o trabalho docente tem uma dimensão afetiva, percebendose com facilidade emoções, afetos, angústias, alegrias e bloqueios afetivos, fatos que tornam o exercício profissional um constante desafio para o patrimônio emocional do professor. Com efeito, o exercício de lecionar pode enriquecer ou empobrecer a dimensão afetiva do ser humano².

Outros componentes, como o idealismo, realização ou não, mediante seu trabalho ou o modo como o realiza, as rotinas institucionais, o processo pedagógico, o que é esperado do seu trabalho pela sociedade, também podem contribuir para a Sobrecarga de Trabalho - ST e ocasionar nos professores diferentes compreensões.

A respeito da ST, algumas instituições dispensam especial atenção às suas causas e prevenção. A Organização Internacional do Trabalho - OIT dedicou algumas das suas tradicionais convenções ao tema, reconhecendo-o como sério problema para a classe trabalhadora em todo o mundo. No Brasil, a Fundação Jorge Figueiredo Duprat FUNDACENTRO e os Ministérios da Saúde, do Trabalho e da Previdência Social repassam os apelos da OIT e emitem parecer sobre a matéria, usando suas ramificações nos estados, municípios e DF para dinamizar a comunicação³.

0 magistério, a segunda maior opção de labor para enfermeiros, é também um mercado que cresceu com a abertura de cursos, fato acelerado nos anos de 2001, 2002 e 2003. No Ceará, no período de 1968 a 2003, os cursos de graduação em Enfermagem chegaram a dezesseis ${ }^{4}$.

0 enfermeiro/professor objeto desta pesquisa exerce sua força de trabalho, evidentemente, no magistério, em diferentes instituições, mas o produto oriundo de seu esforço necessita sempre de mais energia e vem gradativamente perdendo prestígio, embora o número de empregos docentes tenha aumentado. É preciso trabalhar, manter-se com saúde para produzir cada vez mais.

0 mercado de trabalho docente é o público e o privado, atualmente mais o privado do que público, e, em ambos, emergem contradições, de salários, carga horária e proposta de trabalho. Há avanços legislativos, ganhos pelos docentes por meio de suas lutas, mas descumpridos pelos representantes do poder e pelos donos das universidades privadas.

Seja qual for a realidade pública, ou privada, a exploração do trabalho do professor é refletida nos baixos salários, exaustivas normas e rotinas, dificuldades de bibliotecas atualizadas, poucas possibilidades de aperfeiçoamento, jornadas cansativas por ausência de facilidades de exercer 0 trabalho, desvalorização da figura do docente e de suas reivindicações.

Estudiosos consideram que a noção sobre qualidade de vida abrange aspectos da família, do social, do ambiente, no âmbito da cultura, do conforto e do bem-estar ${ }^{5}$. A qualidade de vida é um construto que aufere impor tância na sociedade e, mais particularmente, aos estudiosos da área de Saúde, Psicologia, Sociologia, Antropologia, Educação, dentre outras.

Duas abordagens para medir qualidade de vida foram as de McKenna e Whalley, funcionalistas e baseadas nas necessidades (needs-based). As ideias de Hunt, McKenna e Whalley situam os estudos teóricos de qualidade de vida em dois grandes grupos: os modelos da satisfação e funcionalista ${ }^{6}$.

Além dos modelos teóricos de qualidade de vida, 0 conceito e os construtos de personalidade, emoções e presença de dimensão negativa influenciam a qualidade de vida de tal forma que estudá-las se torna um desafio na atualidade, incorporando aspectos sociais, transculturais, estudos conceituais, metodológicos, psicométricos e estatísticos.

Considerando a qualidade de vida com várias dimensões, neste estudo adotou-se a definição proposta pela Organização Pan-americana de Saúde - "percepção do indivíduo de sua posição na vida no contexto de sua cultura e no sistema de valores em que vive e em relação as suas expectativas, seus padrões e suas preocupações"7:1.

Esta é também a conceituação utilizada pelo grupo Woghol na versão brasileira, ao investir em decisões importantes para que a qualidade de vida seja percebida por profissionais de saúde, pesquisadores e interessados no assunto com clareza conceitual. A Organização Mundial de Saúde adverte para o fato de que, em se tratando de qualidade de vida, a pessoa deve ser o centro da avaliação e que boa saúde não seja considerada como sinônimo de boa qualidade de vida ${ }^{6}$.

Em debate com oito pesquisadores sobre qualidade de vida, foram constatadas as dificuldades de articulação dos indicadores quantitativos e qualitativos para a compreensão do processo, que não deverá permitir sua "ideologização".

A qualidade de vida foi investigada utilizando-se 0 instrumento de avaliação da Organização Mundial de Saúde Whoqol - nos diferentes módulos de Whoqol: Whoqol-100; Whogol- bref, Whogol-hiv; Whogol-srpb e Whoqol-old. 0 domínio geral do Whogol-bref avalia a qualidade de vida global e percepções de saúde geral. É derivado do Whogol-100. As 
questões do Whoqol-brefforam formuladas para uma escala de respostas do tipo Likert nos aspectos: intensidade, capacidade, frequência e avaliação relacionadas aos domínios ${ }^{9}$ a seguir delineados:

No domínio físico, as facetas envolvem a dor e o desconforto, energia e fadiga, sono e descanso, mobilidade, atividades da vida cotidiana, dependência de medicação e de tratamentos e capacidade de trabalho.

Quanto ao domínio psicológico, a qualidade de vida é investigada buscando-se sentimentos positivos, como pensamento, aprendizado, memória e concentração, autoestima, imagem corporal e aparência, e sentimentos negativos, espiritualidade/religião/crenças pessoais.

0 domínio das relações sociais envolveu amigos, parentes, conhecidos e colegas, apoio social e atividade sexual, que são as facetas abordadas.

No domínio do meio ambiente, as facetas investigadas foram segurança física e proteção, ambiente no lar, recursos financeiros, cuidados de saúde e sociais, disponibilidade e qualidade, oportunidades de adquirir novas informações e habilidades, participação e oportunidades de recreação/lazer, ambiente físico e transporte.

0 professor Marcelo Fleck, coordenador do grupo nesta área, validou esse instrumento por meio de amplo estudo realizado no Estado do Rio Grande do Sul em 1998. OWhoqolbref foi criado para avaliar a Qualidade de Vida, sob uma perspectiva internacional, traduzido em 50 idiomas e já foi utilizado em mais de 51 países.

As informações no Scientific Electronic Library Online - SciELO, obtidas em dezembro de 2009, indicavam que, das 74 publicações que informaram ter utilizado o WHOQOL- bref, 70 pesquisaram qualidade de vida em geral e quatro, qualidade de vida do enfermeiro, mas nenhuma delas, a qualidade de vida do enfermeiro docente.

As quatro pesquisas anteriormente indicadas utilizaram o Whoqol-brefe estudaram a qualidade de vida dos acadêmicos de Enfermagem em seis cursos da região Sul do Brasil ${ }^{10}$; investigaram sobre os fatores associados à qualidade de vida de enfermeiras hospitalares chilenas ${ }^{11}$; avaliaram a qualidade de vida dos técnicos e auxiliares de Enfermagem de unidade de terapia intensiva ${ }^{12}$; verificaram a qualidade de vida de acadêmicos de graduação de Enfermagem do primeiro e quarto anos ${ }^{13}$.

Uma pesquisadora da Universidade Nacional da Colômbia com sede em Bogotá realizou a investigação "A Docência: um Risco para a Saúde?" Trata-se de um estudo em que foram pesquisados as atividades, o processo de trabalho, as condições de trabalho, a saúde, os efeitos da carga física do trabalho docente sobre a saúde, o objetosujeito do trabalho docente, os meios, a organização, a divisão do trabalho, as condições de seguridade e os riscos inerentes ao processo da docência. 0 estudo permitiu reconhecer e estabelecer as características do processo de trabalho docente e compará-los com o trabalho em indústrias ${ }^{14}$.

Quanto à saúde dos professores pesquisados, foram encontrados: cansaço, fadiga, alterações osteomusculares, problemas visuais, problemas com a voz, estresse, insatisfação, cefaleias, transtornos gastrointestinais - como úlceras, dispepsias, alterações na eliminação intestinal, no apetite e no sono e problemas de percepção, como falhas ao ler e ao escrever, problemas cardiovasculares, como hipertensão e arritmias. Os dados referentes às condições das tarefas dos docentes encontraram problemas de esforço físico e mental semelhantes aos que são informados nas pesquisas a respeito do trabalho nas indústrias ${ }^{14}$.

No Brasil "as condições de trabalho influenciam no processo de trabalho e contribuem sobremaneira para determinar o processo de saúde-doença dos trabalhadores de enfermagem"15:252.

Então, com suporte na contextualização, destacam-se os questionamentos desta pesquisa: terá o enfermeiro docente disposição para conquistar para si qualidade de vida? Receberá da sociedade meios, facilidades e estímulo para tal? Em razão do exposto objetivou-se verificar a qualidade de vida do enfermeiro docente que trabalha em instituição federal, estadual e privada e comparar os resultados entre os três grupos.

\section{METODOLOGIA}

Um estudo quantitativo do tipo transversal foi realizado de março a maio de 2010, com esteio na ficha do respondente e instrumento de avaliação de qualidade de vida - o Whoqol-brefou abreviado, da Organização Mundial da Saúde, versão em Português com 26 perguntas estruturadas, avaliando diversas facetas, duas das quais sobre a qualidade de vida geral e 24 acerca dos quatro domínios - físico, psicológico, relaç̃es sociais e meio ambiente. ${ }^{16} 0$ questionário foi autoadministrado, assistido pelo entrevistador no local de trabalho dos docentes, cujas respostas referiram-se às duas últimas semanas anteriores ao dia da coleta de dados.

0 cenário envolveu três universidades de uma capital do nordeste em que 38 enfermeiros docentes, com título de doutor, realizavam atividades no ensino de graduação e pósgraduação em Enfermagem. Destes, 18 pertenciam a uma instituição de ensino federal (UF), 14 do ensino estadual (UE) e 6 de uma particular (UP).

Como critérios de inclusão, os professores deveriam estar inseridos em atividades de ensino na graduação e pósgraduação em Enfermagem. Foram excluídos os professores com atividades apenas na graduação.

Na análise dos dados, foram comparados os resultados do Whoqol-bref encontrados nos três grupos (UF, EU e UP) e a interpretação baseada nas investigações que, embora não tenham utilizado o Whogol-bref, tinham como sujeitos enfermeiros docentes.

0 Comitê de Ética e Pesquisa da Universidade Federal do Ceará-COMEPE aprovou e expediu o parecer favorável por meio do Of. $N^{0} 01 / 10$. Os participantes assinaram o Termo de Consentimento Livre e Esclarecido de acordo com as normas da Resolução n0 196/96 17. 


\section{RESULTADOS}

Dos 38 sujeitos, 37 eram do sexo feminino e um do sexo masculino, com faixa etária entre 31 e 62 anos, sendo a média de idade de 46,5 anos. Quanto ao estado civil, seis eram solteiros, 30 casados e dois separados.
Duas são as tabelas que apresentam os resultados do Whoqol-bref. A tabela 1, sobre a qualidade de vida global dos enfermeiros docentes e, a tabela 2, a respeito dos domínios físico, psicológico, relações sociais e meio ambiente, que agregados permitem melhor apreensão dos resultados da escala nos domínios.

Tabela 1 - Qualidade de vida global dos enfermeiros docentes. $n=$ 38 Fortaleza/CE, 2010

\begin{tabular}{|c|c|c|c|c|c|}
\hline \multirow{3}{*}{ Escores } & \multicolumn{3}{|c|}{ Universidade } & \multirow{2}{*}{\multicolumn{2}{|c|}{ Total }} \\
\hline & UF & UE & UP & & \\
\hline & $f$ & $f$ & $f$ & $\mathrm{~F}$ & $\%$ \\
\hline Ruim & 1 & 1 & 0 & 2 & 5,2 \\
\hline Nem ruim nem boa & 4 & 4 & 4 & 12 & 31,6 \\
\hline Boa & 13 & 9 & 2 & 24 & 63,2 \\
\hline Total & 18 & 14 & 6 & 38 & 100 \\
\hline
\end{tabular}

As respostas da Tabela 2 estão relacionadas a cada domínio; entretanto, o n é diferenciado, pois se refere às sete perguntas do domínio físico, seis do domínio psicológico, três do domínio das relações sociais e oito do domínio do meio

ambiente. Ao multiplicar-se pelo número de sujeitos, 18 da UF, 14 da EU e 6 da UP foram obtidos $n=266$ para $01^{\circ}$ domínio; $\mathrm{n}=228$ para $\circ 2^{\circ}, 114$ para $\circ 3^{\circ}$ e 304 para $04^{\circ}$ respectivamente.

Tabela 2 Qualidade de vida dos enfermeiros docentes nos domínios físico,psicológico, relações sociais e meio ambiente. Fortaleza/CE, 2010.

\begin{tabular}{|c|c|c|c|c|c|}
\hline \multirow{3}{*}{ Eccores do donínio fisico $n=266$} & \multicolumn{3}{|c|}{ Ihiersidader } & \multirow{2}{*}{\multicolumn{2}{|c|}{ Totid }} \\
\hline & \multicolumn{2}{|c|}{ IF $\mathbf{U E}$} & \multirow{2}{*}{$\frac{\mathbf{P}}{\mathbf{f}}$} & & \\
\hline & $\mathbf{f}$ & $\mathbf{f}$ & & $\mathbf{f}$ & if \\
\hline Nada, muito rum & 13 & 7 & 9 & 29 & 109 \\
\hline Mito poucolnuim & 15 & 20 & 2 & 37 & 139 \\
\hline Médio, nem ruim nem bơm & 30 & 24 & 5 & 59 & 222 \\
\hline Mito ou bom & 39 & 34 & 14 & 87 & 32,7 \\
\hline Completamente ou Extremamente & 29 & 13 & 12 & 54 & 203 \\
\hline Total & 126 & 98 & 42 & 266 & 100 \\
\hline \multirow{3}{*}{ Escơres do dorírio psicológico $n=228$} & \multicolumn{3}{|c|}{ Uhiersidader } & \multirow{2}{*}{\multicolumn{2}{|c|}{ Total }} \\
\hline & \multicolumn{2}{|c|}{ IF $\mathbf{U E}$} & $\mathbf{U P}$ & & \\
\hline & $\mathbf{f}$ & $\mathbf{F}$ & $\mathbf{f}$ & $\mathbf{f}$ & 8 \\
\hline Nada, muito sđisfeto, nunca & 4 & 3 & 1 & 8 & 3,6 \\
\hline Mito pouco & 12 & 9 & 6 & 27 & 11,8 \\
\hline Mais ou menos innédio & 27 & 32 & 5 & 64 & 280 \\
\hline Bastante,Muto & 42 & 29 & 14 & 85 & 37,3 \\
\hline Extremsmente, Completamente & 23 & 11 & 10 & 44 & 193 \\
\hline Total & 108 & 84 & 36 & 228 & 100 \\
\hline \multirow{3}{*}{$\begin{array}{l}\text { Escores do domínio dæ reações sociass } \\
\qquad n=114\end{array}$} & \multicolumn{3}{|c|}{ Uhiersidates } & \multirow{2}{*}{\multicolumn{2}{|c|}{ Total }} \\
\hline & \multicolumn{2}{|c|}{ IF $\quad$ UE } & $\mathbf{L P}$ & & \\
\hline & $\mathbf{f}$ & $\mathbf{f}$ & $\mathbf{f}$ & $\mathbf{f}$ & 柾 \\
\hline Mito insatisfeito & 1 & 3 & 0 & 4 & 3,0 \\
\hline Insatisfeito & 0 & 2 & 0 & 2 & 1,7 \\
\hline Nem saisfieito inem insatisfeito & 9 & 9 & 2 & 20 & 17,5 \\
\hline Satisfrito & 26 & 20 & 10 & 56 & 49.1 \\
\hline Mito Satisfesto & 18 & 8 & 6 & 32 & 28,7 \\
\hline Total & 54 & 42 & 18 & 114 & 100 \\
\hline \multirow{3}{*}{$\begin{array}{l}\text { Escores do dorínio do meio } \\
\text { ambiente } n=304\end{array}$} & & Essidad & & & \\
\hline & $\mathbf{I F}$ & & $\mathbf{U P}$ & & \\
\hline & $\mathbf{f}$ & $\mathbf{F}$ & $\mathbf{f}$ & $\mathbf{f}$ & 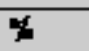 \\
\hline Nadaijmuito insatisfitio & 3 & 0 & 0 & 3 & 1,0 \\
\hline Mito pouco & 14 & 1 & 5 & 20 & 65 \\
\hline Meis ou menos ímédio & 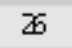 & 36 & 8 & 70 & 23,1 \\
\hline Bastanteimuito & 66 & 48 & 20 & 134 & 44,1 \\
\hline Extremementejconpletamente & 35 & 27 & 15 & $\pi$ & 25,3 \\
\hline Total & 114 & 112 & 48 & 304 & 100 \\
\hline
\end{tabular}




\section{DISCUSSÃO}

Ao comparar os três grupos na Tabela 1, somente os professores da universidade privada têm minoria no escore "bom", enquanto os dois grupos apresentaram o maior percentual.

$\mathrm{Na}$ Tabela 2, os enfermeiros docentes contemplaram todos os escores do domínio físico. Vale salientar que muito bom, bom e completamente corresponderam a $53 \%$ das respostas dos três grupos, enquanto para os outros escores a soma foi de $47 \%$ das respostas. Estes últimos referem-se aos escores de pior qualidade de vida, próximos daqueles encontrados no estudo com enfermeiras chilenas com média em torno de $54,56^{11}$. É importante ressaltar que as enfermeiras trabalham no hospital em escala noturna, e o cansaço pela especificidade do processo de trabalho envolve grande parte das facetas deste domínio. No caso das enfermeiras docentes, a sobrecarga de trabalho está relacionada às atividades de ensino, pesquisa, extensão e administração .

Quanto ao domínio psicológico, 56,6\% referiram estarem bastante/muito satisfeitos, e completamente. Quanto aos escores "nada", "muito insatisfeito" e "insatisfeito", corresponderam a $43,4 \%$. Há semelhança nas respostas dos enfermeiros docentes relacionadas ao domínio físico e psicológico.

No domínio das relações sociais, para os escores "satisfeito" e "muito satisfeito" estão 77,8\% das respostas, e para "muito insatisfeito", "insatisfeito" e "nem satisfeito nem insatisfeito", 22,2\%.

Em relação ao domínio do meio ambiente para os escores "bastante", "muito" e "satisfeito" estão 69,4\% das respostas e $30,6 \%$ a nada, muito insatisfeito, insatisfeito e nem satisfeito nem insatisfeito.

Na análise da Tabela 2, observou-se que, nos domínios físico e psicológico, a soma dos percentuais dos escores nada/ muito ruim/muito insatisfeito, muito pouco/insatisfeito, mais ou menos/médio não exibiu diferenças relevantes, visto que, no primeiro, foi de $47 \%$ e no segundo $43,4 \%$; entretanto, para os domínios das relações sociais e do meio ambiente, foram $22,2 \%$ e $30,6 \%$, respectivamente.

A soma dos percentuais dos escores muito ou bom, bastante/muito satisfeito e extremamente/completamente correspondeu a 53\% no domínio físico, 56,6\% no psicológico, $77,8 \%$ nas relações sociais e $69,4 \%$ no meio ambiente. Quanto aos percentuais dos escores nada/nunca, muito ruim, médio, nem ruim nem bom, muito pouco, ruim, muito insatisfeito/ insatisfeito, nem satisfeito/nem insatisfeito corresponderam a $47 \%, 43,4 \%, 22,2 \%$ e $30,6 \%$, respectivamente.

No âmbito de dois dos domínios, o físico, com $53 \%$ e o psicológico com $56,6 \%$, nota-se que os percentuais dos escores demonstraram discretas variações, mas no que se refere ao das relações sociais e o do meio ambiente, constatou-se uma diferença considerável nos escores do primeiro com $55 \%$ e do segundo com $38 \%$.
Avaliar a qualidade de vida na perspectiva dos quatro domínios permitiu conhecer os aspectos vulneráveis dos três grupos no conjunto das diversidades das facetas que, de certa forma, podem sinalizar para os enfermeiros docentes a possibilidade de modificações adequadas às expectativas pessoais.

\section{CONCLUSÃO}

A realização do estudo com uso do Whogol-bref, mesmo com amostra reduzida, permitiu comparar a qualidade de vida entre os docentes das três universidades no âmbito dos quatro domínios, ensejando, assim, a consecução do objetivo traçado,

A metodologia utilizada revelou-se adequada para a obtenção dos principais achados apresentados, destacando a relevância do estudo e potencial campo de pesquisa em Enfermagem em diversos outros aspectos.

Verificou-se que a qualidade de vida global dos enfermeiros docentes foi considerada boa pelos três grupos, e, quanto aos escores mais negativos e mais positivos, constatou-se que apresentaram diferenças relevantes nos dois últimos domínios - o das relações sociais e do meio ambiente.

Os resultados apontam para a necessidade de ampliar a discussão sobre a qualidade de vida dos docentes de Enfermagem, assim como o conhecimento da satisfação no exercício da atividade de ensino, considerando os possíveis descontentamentos decorrentes da prática de ensinar e seu impacto sobre a vida dos exercentes.

Os resultados trazem reflexões também sobre 0 desenvolvimento da profissão, tendo como foco a característica privativa das ações do enfermeiro, que está voltada para a prestação do cuidado à sociedade em condições de saúde e doença em todo ciclo vital.

\section{REFERÊNCIAS}

1. Secco IAO, Robazzi MLCC, Shimizu DS, Rubio MMS. Acidentes de trabalho típicos envolvendo trabalhadores de hospital universitário da região sul do Brasil: epidemiologia e prevenção. Rev Latino-Am Enfermagem. 2008; 16(5): 824-831.

2. Tardif M. Saberes docentes e formação profissional. Petrópolis(RJ) : Vozes; 2002.

3. Ministério do Trabalho (BR). Boletim Informativo 2001. Brasilia(DF): Ministério do Trabalho: 2001.

4.Ministerio da Saude (BR). Sistema Nacional de Avaliação do Ensino Superior. Avaliação de Cursos de Graduação do Sistema Nacional de Avaliação da Educação Superior - SINAES para os cursos de graduação em Medicina, Enfermagem e Odontologia. [citado 2010 out 10].

Disponível em: http://wwwportal.mec.gov. 
5. Minayo MCS, Hartz MA, Buss PM. Qualidade de vida e saúde: um debate necessário. Cienc Saude Colet .2000; 5(1): 7-18

6. Fleck MPA. A avaliação de qualidade de vida: guia para profissionais de saúde. Porto Alegre: Artmed; 2008.

7. The WHOQOL Group. The world health organization of life assessment (WHOQOL): position paper from the World Health Organization. Soc Sci Med .1995; 41: 1403-09.

8. Costa MS. Estilos de vida saudável: novas referências nas práticas de saúde [dissertação de mestrado]; Fortaleza:Faculdade de Farmácia, Odontologia e Enfermagem/UFC;2004.

9. Fleck MPA. Projeto desenvolvido no Brasil pelo grupo de estudos em qualidade de vida. [citado 2010 out 22]. Disponível em: http:// www.ufrgs.br .

10. Saupe R, Nietche EA, Cestari ME, Giorgi MDM. Qualidade de vida dos acadêmicos de enfermagem. Rev Latino-Am Enfermagem. 2004; 12(4): 641.

11. Andrades LB, Valenzuela SS. Fatores associados à qualidade de vida de enfermeiras hospitalares chilenas. Rev Latino-Am Enfermagem. 2007; 15(3): 480-86.

12. Paschoa S, ZaneiSSV, Whitaker IY. Qualidade de vida dos trabalhadores de enfermagem de unidades de terapia intensiva. Acta Paul Enferm. 2007; 20(3), p.305-310.

13. Eurich RB, Kluthcovsky ACGC. Avaliação da qualidade de vida de acadêmicos de graduação em Enfermagem do primeiro ao quarto anos. Rev Psiquiatr. 2008; 30(3): 211-20.

14. Lozada MAL. La docencia: un riesgo para la salud? Avances en Enfermeria. 2005; 23(1): 18-30.

15. Mauro MYC, Paz AF, Mauro CCC, Pinheiro MAS. Condições de trabalho da enfermagem nas enfermarias de um hospital universitário. Esc Anna Nery . [on-line]. 2010 jun [citado 2011 mai 20]; 14(2): Disponível: http://www.scielo.br.

16. Organização Mundial de Saúde- OMS. WHOQOL, abreviado, versão em português. [citado 2010 out 24]. Disponível em:<www.ufrgs.br/ psiq/whogol>.

17. Ministério da Saúde (BR). Conselho Nacional de Saúde. Comitê Nacional de Ética em Pesquisa em Seres Humanos. Resolução n.196, de 10 de outubro de 1996: diretrizes e normas regulamentadoras de pesquisa envolvendo seres humanos. Brasília (DF); 1997. 